\title{
TIME-DEPENDENT LIPSCHITZ ATTRACTORS FOR NON-SEMIGROUP EVOLUTION PROCESSES
}

\author{
MIHAI TURINICI \\ (Received 26 November 1982; revised 15 July 1983) \\ Communicated by J. F. Price
}

\begin{abstract}
A maximality principle on quasi-ordered pseudo-metric spaces is used to obtain a number of Lipschitz attraction results for non-semigroup evolution processes with respect to time-dependent families. As particular cases, a multivalued version of Dieudonné's means value theorem and the Kirk-Ray lipschitzianness test are derived.

1980 Mathematics subject classification (Amer. Math. Soc.): primary 54 F 05, 54 H 20; secondary 26 A 24.

Key words and phrases: maximal element, evolution process, function-attractive time-dependent family, mean value theorem, lipschitzianness test.
\end{abstract}

\section{Introduction}

An important question concerning a wide class of evolution processes acting on (pseudo-) metric structures is that of determining sufficient "local" conditions in order that a "global" attraction property be obtained (the word "attraction", not reducible to that used by Bhatia and Szegö [2, Ch. V, §5], being made precise by the context of the problem). As basic results in the semigroup case, one must quote the 1970 Brézis' invariance theorem [4] proved by a differential inequality procedure, and the 1976 Brézis-Browder attraction test [5] obtained by the use of a general principle on ordered sets. A non-semigroup counterpart of these contributions was performed in the 1981 author's paper [21], through a metrical Zorn argument. It is the main intention of the present exposition to get a 
time-dependent extension of these developments, the basic tool of our investigations being a pseudo-metric maximality principle appearing as a common extension of the above quoted Brézis-Browder and author's ones. As direct applications, a "multivalued" version of Dieudonné's mean value theorem [11, Ch. VIII, §5] and Kirk-Ray lipschitzianness test [16] are given; a number of subsequent extensions of this last result (including a "multivalued" variant of Clarke's fixed point principle [10]) will be discussed elsewhere.

\section{A pseudo-metric maximality principle}

Let $X$ be a nonempty set and let $\leqslant$ be a quasi-ordering (that is, a reflexive and transitive relation) on $X$. A sequence $\left(x_{n} ; n \in \mathbf{N}\right)$ in $X$ will be said to be increasing if $x_{i} \leqslant x_{j}$ whenever $i \leqslant j$, and bounded above provided that $x_{n} \leqslant y, n \in \mathrm{N}$, for some $y$ in $X$ called in this circumstance an upper bound of the considered sequence. Furthermore, $d$ being a pseudo-metric (that is, a non-sufficient metric) on $X$, and $Y$ a subset of $X$, let us term the element $z$ in $Y,(d, Y)$-maximal when $z \leqslant y \in Y$ implies $d(z, y)=0$. Concerning this notion, the following maximality principle will play a central role in the sequel.

THEOREM 1 ([27]). Suppose the quasi-ordered pseudo-metric space $(X, d, \leqslant)$ and the subset $Y$ of $X$ are such that

(i) any increasing sequence in $Y$ is d-Cauchy (in $X$ )

(ii) any increasing $d$-Cauchy sequence in $Y$ is bounded above (in $Y$ ).

Then, to every $x$ in $Y$ there corresponds $a(d, Y)$-maximal element $z$ in $Y$ with $x \leqslant z$.

As already noted in the above quoted paper, when $d$ is taken of the form

$$
d(x, y)=|\varphi(x)-\varphi(y)|, \quad x, y \in X,
$$

$\varphi$ being a function from $X$ into $R$ whose restriction to $Y$ is decreasing and bounded from below, condition (i) will be clearly fulfilled so that, under the acceptance of (ii), Theorem 1 is applicable to these elements; of course, its corresponding statement [to any $x$ in $Y$ there corresponds a $z \in Y$ with (a) $x \leqslant z$, (b) $z \leqslant y \in Y$ implies $\varphi(z)=\varphi(y)]$ is nothing but the Brézis-Browder ordering principle [5]. Particularly, when $X$ is endowed with a uniformity $\mathscr{U}$ satisfying

(ii) for every $U$ in $q$ there exists $\varepsilon>0$ such that, $x, y \in Y, x \leqslant y$ and $|\varphi(x)-\varphi(y)|<\varepsilon$ imply $(x, y) \in U$

(ii) ${ }_{2}$ any increasing $\mathscr{Q}$-Cauchy sequence in $Y$ is bounded above (in $Y$ ) condition (ii) will evidently take place and the appropriate statement of Theorem 1 [to any $x$ in $Y$ there corresponds a $z \in Y$ with (a) $x \leqslant z$, (b)' $z \leqslant y \in Y$ implies 
$(z, y) \in U$ for all $U$ in $\mathscr{U}$ ] coincides in fact with a related Brondsted's one [6] proved by a direct Zorn procedure. Moreover, it was shown these developments may be regarded as a generalization of the variational type Ekeland's result [12] (see also Turinici [21]) or equivalently—after Brondsted's pattern [7]—of the fixed point Caristi-Kirk theorem [9, 15] (see in this direction Kasahara [14], Browder [8], Wong [28], Pasicki [19], Siegel [20] for a number of related viewpoints concerning this problem) so, the result under consideration extends all these contributions. Finally, a slight generalization of Theorem 1 may be obtained if, instead of a single pseudo-metric $d$, a denumerable family $D$ of such elements would be taken; an explicit statement of this assertion, together with an application to nonlinear mapping theory may be found in Turinici [25].

\section{The main results}

In what follows, a precise statement of the considerations exposed in the introductory part of the paper will be performed. Let $(V, d)$ be a complete pseudo-metric space and let $J$ be a real interval. By convention, any mapping $(t, v) \mapsto S(t, v)=S(t) v$ from $J \times V$ into $V$ will be termed a $J$-evolution process (on $V$ ); also, a mapping $t \mapsto F(t)=F_{t}$ from $J$ into $2^{V}$ (the family of all non-empty subsets of $V$ ) will be called a $J$-family (in $V$ ). At the same time, for any mapping $S$ defined as above and any $t \in J, W \in 2^{V}, S(t) W$ will indicate the subset of all $S(t) w, w \in W$. Finally, given the couple $X, Y$ in $2^{V}$, by $D_{1}(X, Y)$ $\left(D_{2}(X, Y)\right)$ we shall denote the supremum over $x \in X(y \in Y)$ of the expressions $d(x, Y)=\inf \{d(x, y) ; y \in Y\}(d(X, y)=\inf \{d(x, y) ; x \in X\})$; clearly, $D=$ $\max \left(D_{1}, D_{2}\right)$ is nothing but the (generalized) Hausdorff pseudo-metric in $2^{V}$. Finally, given the increasing function $h$ from $J$ into $R$, let us term the $J$-family $F$, $D_{1}\left(D_{2}\right)$-globally $h$-attractive with respect to the $J$-evolution process $S$, provided that

$$
\begin{aligned}
& D_{1}\left(S(a) F_{a}, S(b) F_{b}\right) \leqslant h(b)-h(a), \quad a, b \in J, a<b, \\
& \left(D_{2}\left(S(a) F_{a}, S(b) F_{b}\right) \leqslant h(b)-h(a), \quad a, b \in J, a<b\right) .
\end{aligned}
$$

Concerning this notion, the first main result of the present paper is

THEOREM 2. Suppose the couple ( $S, F)$ possesses the strong closedness property

(iii) for any increasing (decreasing) sequence $\left(t_{n} ; n \in N\right)$ in $J$ and any sequence $\left(v_{n} ; n \in N\right)$ in $V$ with $t_{n} \rightarrow t \in J, v_{n} \in F\left(t_{n}\right), n \in \mathbf{N}$, and $S\left(t_{n}\right) v_{n} \rightarrow w \in V$, there exists $v \in F_{t}$ with $S(t) v=w$

and let the strictly increasing function $h: J \rightarrow \mathbf{R}$ be such that, a denumerable subset 
A of $\mathbf{R}$ may be found with

(iv) for any $s$ in $J \backslash A$ not identical with the eventual right(left) end-point of $J$ we have

$$
\begin{gathered}
\liminf _{r \rightarrow s+} d\left(S(s) v, S(r) F_{r}\right) /(h(r)-h(s)) \leqslant 1, \quad v \in F_{s}, \\
\left(\liminf _{r \rightarrow s^{-}} d\left(S(s) v, S(r) F_{r}\right) /(h(s)-h(r)) \leqslant 1, \quad v \in F_{s}\right),
\end{gathered}
$$

(v) for any $s$ in $J \cap A$ not identical with the eventual right (left) end-point of $J$ we have

$$
\begin{gathered}
\liminf _{r \rightarrow s^{+}} d\left(S(s) v, S(r) F_{r}\right) \leqslant h(s+0)-h(s), \quad v \in F_{s}, \\
\left(\liminf _{r \rightarrow s_{-}} d\left(S(s) v, S(r) F_{r}\right) \leqslant h(s)-h(s-0), \quad v \in F_{s}\right) .
\end{gathered}
$$

Then, $F$ is necessarily $D_{1}\left(D_{2}\right)$-globally h-attractive with respect to $S$.

Proof. Let $n \mapsto a_{n}$ be a bijection from $\mathbf{N}$ to $A$ and let $g: \mathbf{R} \rightarrow \mathbf{R}$ be defined, for every $t \in \mathbf{R}$, by

$$
\begin{aligned}
g(t) & =\sum\left\{1 / 2^{n} ; a_{n}<t\left(a_{n} \leqslant t\right)\right\}, & & \text { if }\left\{n \in N ; a_{n}<t\left(a_{n} \leqslant t\right)\right\} \text { is not empty, } \\
& =0, & & \text { otherwise; }
\end{aligned}
$$

evidently, $g$ is increasing and satisfies $0 \leqslant g(t) \leqslant 1, t \in \mathbf{R}$, as well as

$$
\begin{array}{ll}
g(t)-g\left(a_{n}\right) \geqslant 1 / 2^{n}, & \text { whenever } t>a_{n}, \\
\left(g\left(a_{n}\right)-g(t) \geqslant 1 / 2^{n},\right. & \text { whenever } \left.t<a_{n}\right) .
\end{array}
$$

Now, $\gamma>1$ and $\varepsilon>0$ being arbitrary fixed, let us define the function $f: J \rightarrow \mathbf{R}$ by

$$
f(t)=\gamma h(t)+\varepsilon g(t), \quad t \in J,
$$

and let $X$ denote the graph of the $J$-family $F$ (the subset of all pairs $(t, v)$ in $J \times V$ with $v \in F_{t}$ ) endowed with the pseudo-metric

$$
e((t, u),(s, v))=|t-s|+d(S(t) u, S(s) v), \quad(t, u),(s, v) \in X,
$$

and with the quasi-ordering

$$
\left\{\begin{aligned}
&(t, u) \leqslant(s, v) \quad \text { if and only if } t \leqslant s(t \geqslant s), \text { and } d(S(t) u, S(s) v) \\
& \leqslant f(s)-f(t)(d(S(t) u, S(s) v) \leqslant f(t)-f(s)) .
\end{aligned}\right.
$$

Furthermore, given $a, b$ in $J$ with $a<b$, let $Y$ denote the subset of all $(t, v)$ in $X$ with $a \leqslant t \leqslant b$, and let $\left(\left(t_{n}, v_{n}\right) ; n \in \mathrm{N}\right)$ be an increasing (modulo $\leqslant$ ) sequence in 
$Y$, that is

$$
\begin{array}{r}
t_{n} \leqslant t_{m}, d\left(S\left(t_{n}\right) v_{n}, S\left(t_{m}\right) v_{m}\right) \leqslant f\left(t_{m}\right)-f\left(t_{n}\right), \quad n \leqslant m, \\
\left(t_{n} \geqslant t_{m}, d\left(S\left(t_{n}\right) v_{n}, S\left(t_{m}\right) v_{m}\right) \leqslant f\left(t_{n}\right)-f\left(t_{m}\right), \quad n \leqslant m\right) .
\end{array}
$$

From the first part of this inequality, $\left(t_{n} ; n \in \mathbf{N}\right)$ appears as an increasing (decreasing) sequence in $[a, b]$ so that, necessarily, $t_{n} \rightarrow t$ for some $t$ in $[a, b]$; also, from the second part of (3), $\left(S\left(t_{n}\right) v_{n} ; n \in \mathbf{N}\right)$ is a $d$-Cauchy sequence in $V$ and therefore, by completeness, $S\left(t_{n}\right) v_{n} \rightarrow w$ for some $w$ in $V$. It immediately follows by these properties $\left(\left(t_{n}, v_{n}\right) ; n \in \mathbf{N}\right)$ is an $e$-Cauchy sequence in $X$ and moreover (by the strong closedness property (iii)) that a $v \in F_{t}$ may be found with $S(t) v=w$; consequently, performing the limit as $m \rightarrow \infty$ in (3), we get

$$
\begin{gathered}
t_{n} \leqslant t, d\left(S\left(t_{n}\right) v_{n}, S(t) v\right) \leqslant f(t)-f\left(t_{n}\right), \quad n \in \mathbf{N}, \\
\left(t_{n} \geqslant t, d\left(S\left(t_{n}\right) v_{n}, S(t) v\right) \leqslant f\left(t_{n}\right)-f(t), \quad n \in \mathbf{N}\right),
\end{gathered}
$$

proving $(t, v) \in Y$ is an upper bound of the considered sequence. By Theorem 1 we conclude that, given $(a, u)((b, u))$ in $Y$ there exists a $(e, Y)$-maximal element $(s, v)$ in $Y$ with $(a, u) \leqslant(s, v)((b, u) \leqslant(s, v))$. Suppose $s<b(s>a)$. For any $(r, w)$ in $Y$ with $s<r \leqslant b(a \leqslant r<s)$ we necessarily have (by the definition of our quasi-ordering)

$$
\begin{gathered}
d(S(s) v, S(r) w)>f(r)-f(s), \quad w \in F_{r}, \\
\left(d(S(s) v, S(r) w)>f(s)-f(r), \quad w \in F_{r}\right),
\end{gathered}
$$

that is,

$$
\begin{array}{ll}
d(S(s) v, S(r) w)>\gamma(h(r)-h(s))+\varepsilon(g(r)-g(s)), & s<r \leqslant b, w \in F_{r}, \\
(d(S(s) v, S(r) w)>\gamma(h(s)-h(r))+\varepsilon(g(s)-g(r)), & \left.a \leqslant r<s, w \in F_{r}\right) .
\end{array}
$$

Now, two cases are open before us: either $s$ belongs to $J \backslash A$ or $s$ belongs to $J \cap A$.

Case 1. $s \in J \backslash A$. Then, as an immediate consequence of (4)

$$
\begin{array}{r}
d\left(S(s) v, S(r) F_{r}\right) \geqslant \gamma(h(r)-h(s)), \quad s<r \leqslant b, \\
\left(d\left(S(s) v, S(r) F_{r}\right) \geqslant \gamma(h(s)-h(r)), \quad a \leqslant r<s\right),
\end{array}
$$

so, dividing by $h(r)-h(s)$ (by $h(s)-h(r)$ ) and taking liminf as $r \rightarrow s+$ (as $r \rightarrow s-$ ) we obtain a contradiction to (iv). 
Case 2. $s \in J \cap A$ that is, $s=a_{n}$ for some $n \in \mathbf{N}$. Then, as another consequence of (4) we get (by (2))

$$
\begin{aligned}
d\left(S(s) v, S(r) F_{r}\right) \geqslant \gamma(h(s+0)-h(s))+\varepsilon / 2^{n}, & s<r \leqslant b, \\
\left(d\left(S(s) v, S(r) F_{r}\right) \geqslant \gamma(h(s)-h(s-0))+\varepsilon / 2,\right. & a \leqslant r<s),
\end{aligned}
$$

in which situation, passing to liminf as $r \rightarrow s+$ (as $r \rightarrow s-$ ), (v) will be contradicted.

From this discussion it is clear that we are forced to accept the possibility $s=\boldsymbol{b}$ $(s=a)$. But then, relation $(a, u) \leqslant(b, v)((b, u) \leqslant(a, v))$ gives immediately

$$
\begin{gathered}
d\left(S(a) u, S(b) F_{b}\right) \leqslant \gamma(h(b)-h(a))+\varepsilon(g(b)-g(a)), \\
\left(d\left(S(a) F_{a}, S(b) u\right) \leqslant \gamma(h(b)-h(a))+\varepsilon(g(b)-g(a))\right),
\end{gathered}
$$

and therefore the result follows, because $\gamma>1, \varepsilon>0$ and $u \in F_{a}\left(F_{b}\right)$ were arbitrary.

Let $(V, d)$ and $J$ be endowed with their previous meaning. We shall say the increasing function $k: J \rightarrow R$ is $i$-admissible (d-admissible) with respect to the $J$-family $F$ provided that the subset $F_{b}(u, k(b)-k(a))\left(F_{a}(u, k(b)-k(a))\right)$ of all $v \in F_{b}\left(F_{a}\right)$ with $d(u, v) \leqslant k(b)-k(a)$ is not empty, for any couple $a, b \in J$, $a<b$ and any element $u \in F_{a}\left(F_{b}\right)$. Furthermore, calling the $J$-family $F, i$-normal (d-normal) when the class $A_{i}(F)\left(A_{d}(F)\right)$ of all $i$-admissible (d-admissible) functions $k: J \rightarrow R$ is not empty, let us indicate, for any function $k \in A_{i}(F)$ $\left(A_{d}(F)\right)$, by $F_{b}\left(F_{a}, k(b)-k(a)\right)\left(F_{a}\left(F_{b}, k(b)-k(a)\right)\right)$ the union of the subsets $F_{b}(u, k(b)-k(a))\left(F_{a}(u, k(b)-k(a))\right)$ with $u \in F_{a}\left(F_{b}\right)$ and, given the increasing function $h: J \rightarrow R$, let us term the $J$-family $F$, $k$-sectionally $D_{1}\left(D_{2}\right)$-globally $h$-attractive with respect to the $J$-evolution process $S$ provided that

$$
\begin{array}{r}
D_{1}\left(S(a) F_{a}, S(b) F_{b}\left(F_{a}, k(b)-k(a)\right)\right) \leqslant h(b)-h(a), \quad a, b \in J, a<b, \\
\left(D_{2}\left(S(a) F_{a}\left(F_{b}, k(b)-k(a)\right), S(b) F_{b}\right) \leqslant h(b)-h(a), \quad a, b \in J, a<b\right) .
\end{array}
$$

Under these conventions, the second main result of the paper may be stated as follows.

THEOREM 3. Suppose the J-family $F$ are such that the couple $(S, F)$ possesses the weak closedness property

for any increasing (decreasing) sequence $\left(t_{n} ; n \in \mathbf{N}\right)$ in $J$ and

(iii) ${ }^{\prime} \quad$ any sequence $\left(v_{n} ; n \in \mathbf{N}\right)$ in $V$ with $v_{n} \in F\left(t_{n}\right), n \in \mathbf{N}$, and $t_{n} \rightarrow t \in J, v_{n} \rightarrow v \in V, S\left(t_{n}\right) v_{n} \rightarrow w \in V$, we have $v \in F_{t}$ and $S(t) v=w$ 
and let the i-admissible (d-admissible) function $k: J \rightarrow \mathbf{R}$ and the strictly increasing function $h: J \rightarrow \mathbf{R}$ be such that, a denumerable subset $A$ of $\mathbf{R}$ may be found with

(iv)' for any $s$ in $J \backslash A$ not identical with the eventual right (left) end-point of $J$ we have

$$
\begin{gathered}
\liminf _{r \rightarrow s^{+}} d\left(S(s) v, S(r) F_{r}(v, k(r)-k(s))\right) /(h(r)-h(s)) \leqslant 1, \quad v \in F_{s}, \\
\left(\liminf _{r \rightarrow s^{-}} d\left(S(s) v, S(r) F_{r}(v, k(s)-k(r))\right) /(h(s)-h(r)) \leqslant 1, \quad v \in F_{s}\right),
\end{gathered}
$$

(v)' for any $\sin J \cap A$ not identical with the eventual right (left) end-point of $J$ we have

$$
\begin{array}{cc}
\liminf _{r \rightarrow s^{+}} d\left(S(s) v, S(r) F_{r}(v, k(r)-k(s))\right) \leqslant h(s+0)-h(s), & v \in F_{s}, \\
\left(\liminf _{r \rightarrow s^{-}} d\left(S(s) v, S(r) F_{r}(v, k(s)-k(r))\right) \leqslant h(s)-h(s-0),\right. & \left.v \in F_{s}\right) .
\end{array}
$$

Then, $F$ is necessarily $k$-sectionally $D_{1}\left(D_{2}\right)$-globally h-attractive with respect to $S$.

Proof. Let the functions $g$ and $f$ be defined as in the above theorem; also, let $X$ denote the graph of the $J$-family $F$, endowed with the pseudo-metric

$$
e((t, u),(s, v))=|t-s|+d(u, v),(t, u),(s, v) \in X
$$

as well as with the quasi-ordering

$$
\left\{\begin{array}{l}
(t, u) \leqslant(s, v) \text { if and only if } t \leqslant s(t \geqslant s), \\
d(u, v) \leqslant k(s)-k(t)(d(u, v) \geqslant k(t)-k(s)) \text { and } \\
d(S(t) u, S(s) v) \leqslant f(s)-f(t)(d(S(t) u, S(s) v) \leqslant f(t)-f(s))
\end{array}\right.
$$

and by $Y$ the subset of all $(t, v)$ in $X$ with $a \leqslant t \leqslant b$. Suppose $\left(\left(t_{n}, v_{n}\right) ; n \in \mathbf{N}\right)$ is an increasing (module $\leqslant$ ) sequence in $Y$, that is

$$
\begin{aligned}
& t_{n} \leqslant t_{m}, \quad d\left(v_{n}, v_{m}\right) \leqslant k\left(t_{m}\right)-k\left(t_{n}\right), d\left(S\left(t_{n}\right) v_{n}, S\left(t_{m}\right) v_{m}\right) \\
& \leqslant f\left(t_{m}\right)-f\left(t_{n}\right), \quad n \leqslant m, \\
&\left(t_{n} \geqslant t_{m}, d\left(v_{n}, v_{m}\right) \leqslant k\left(t_{n}\right)-k\left(t_{m}\right),\right. d\left(S\left(t_{n}\right) v_{n}, S\left(t_{m}\right) v_{m}\right) \\
&\left.\leqslant f\left(t_{n}\right)-f\left(t_{m}\right), \quad n \leqslant m\right) .
\end{aligned}
$$

From the first and the second part of this inequality, it immediately follows, by completeness, $t_{n} \rightarrow t$ and $v_{n} \rightarrow v$ for some $t \in J, v \in V$ (hence, a fortiori, that (i) will be satisfied); moreover, by the last part of (3)' we derive (again by completeness) $S\left(t_{n}\right) v_{n} \rightarrow w$ for some $w \in V$ so (by the weak closedness hypothesis (iii)') $v \in F_{t}$ and $S(t) v=w$ in which case, passing to limit as $m \rightarrow \infty$ in (3)', (ii) will be satisfied too and Theorem 1 applies. The remaining part of the proof is the same as in the above result except that we have to replace $F_{r}$ by $F_{r}(v, k(r)-k(s))$ (by $\left.F_{r}(v, k(s)-k(r))\right)$ in the analysis of the corresponding cases 1 and 2 . Finally, it 
suffices to observe relation $(a, u) \leqslant(b, v)((b, u) \leqslant(a, v))$ yields immediately

$$
\begin{aligned}
& d\left(S(a) u, S(b) F_{b}\left(F_{a}, k(b)-k(a)\right)\right) \leqslant \gamma(h(b)-h(a))+\varepsilon(g(b)-g(a)), \\
& \left(d\left(S(a) F_{a}\left(F_{b}, k(b)-k(a)\right), S(b) u\right) \leqslant \gamma(h(b)-h(a))+\varepsilon(g(b)-g(a))\right)
\end{aligned}
$$

and this will complete the proof, because $\gamma>1, \varepsilon>0$ and $u \in F_{a}\left(F_{b}\right)$ were arbitrary.

A close analysis of the assumption (iv) ((iv)') essentially involved into the first (second) main result shows it may be expressed under a more symmetric form with respect to its component elements. To do this, let us introduce, for any function $f: J \rightarrow \mathbf{R}$ and any $t \in J$ not identical with the eventual right (left) end-point of $J$ : the notation $D_{(+)}^{+} f(t)\left(D_{(-)}^{-} f(t)\right)$ to mean limsup/inf as $s \rightarrow t+$ (as $s \rightarrow t-$ ) of the quotient $(f(s)-f(t)) /(s-t)$; of course, this expression is nothing but the upper/lower right (left) Dini derivative of the function $f$ at the point $t$. Now, as a useful "combined" variant of these results, we have

THEOREM 4. Suppose in the first (second) statement the function $h: J \rightarrow \mathbf{R}$ is only increasing and that assumption (iv) (respectively, (iv)') is substituted by

(vi) for any $s$ in $J \backslash A$ not identical with the eventual right (left) end-point of $J$ we have

$$
\begin{array}{cc}
+\infty \neq \lim \sup _{r \rightarrow s+} / \inf d\left(S(s) v, S(r) F_{r}\right) /(r-s) \leqslant D_{(+)}^{+} h(s), & v \in F_{s}, \\
\left(+\infty \neq \lim \sup _{r \rightarrow s^{-}} \inf d\left(S(s) v, S(r) F_{r}\right) /(s-r) \leqslant D_{(-)}^{-} h(s),\right. & \left.v \in F_{s}\right),
\end{array}
$$

[respectively

(vi)' for any $s$ in $J \backslash A$ not identical with the eventual right (left) end-point of $J$ we have

$$
\begin{array}{r}
+\infty \neq \limsup / \inf d\left(S(s) v, S(r) F_{r}(v, k(r)-k(s))\right) /(r-s) \leqslant D_{(+)}^{+} h(s), \\
v \in F_{s}, \\
\left(+\infty \neq \limsup _{r \rightarrow s^{-}} \inf d\left(S(s) v, S(r) F_{r}(v, k(s)-k(r))\right) /(s-r) \leqslant D_{(-)}^{-} h(s),\right. \\
\left.\left.v \in F_{s}\right)\right] .
\end{array}
$$

Then, the conclusion of the first (second) main result will remain unchanged.

Proof. Let $f: J \rightarrow \mathbf{R}$ be defined as

$$
f(t)=\gamma h(t)+\varepsilon g(t)+\delta t, \quad t \in J,
$$


$\gamma>1, \varepsilon>0$ and $\delta>0$ being arbitrary fixed. Repeating - under this choice of the function $f$-the arguments we already exposed, it is not hard to see the inequalities of the Case 1 yield the evaluations

$$
\begin{array}{r}
d\left(S(s) v, S(r) F_{r}\right) \geqslant \gamma(h(r)-h(s))+\delta(r-s), \quad s<r \leqslant b, \\
\left(d\left(S(s) v, S(r) F_{r}\right) \geqslant \gamma(h(s)-h(r))+\delta(s-r), \quad a \leqslant r<s\right),
\end{array}
$$

[respectively

$$
\begin{array}{r}
d\left(S(s) v, S(r) F_{r}(v, k(r)-k(s))\right) \geqslant \gamma(h(r)-h(s))+\delta(r-s), \\
s<r \leqslant b, \\
\left(d\left(S(s) v, S(r) F_{r}(v, k(s)-k(r))\right) \geqslant \gamma(h(s)-h(r))+\delta(s-r),\right. \\
a \leqslant r<s)],
\end{array}
$$

so, if we divide by $r-s$ (by $s-r$ ) and take limsup/inf as $r \rightarrow s+($ as $r \rightarrow s-$ ) in both sides, condition (vi) (respectively, (vi)') will be contradicted. This completes the proof.

Concerning the other elements implicated into these results, some remarks are in order. Firstly, it is clear that, in case the $J$-family $F$ is order-usc in the following sense

(vii) $\quad t_{n} \rightarrow t \in J$ and any sequence $\left(v_{n} ; n \in \mathbf{N}\right)$ in $V$ with $v_{n} \rightarrow v \in V$, relations $v_{n} \in F\left(t_{n}\right), n \in \mathbf{N}$, imply $v \in F_{t}$

a sufficient condition for (iii) is

if the increasing (decreasing) sequence $\left(t_{n} ; n \in \mathbf{N}\right)$ in $J$ with (viii) $\quad t_{n} \rightarrow t \in J$ and the sequence $\left(v_{n} ; n \in N\right)$ in $V$ are such that $S\left(t_{n}\right) v_{n} \rightarrow w \in V$ then $V_{n} \rightarrow V$ and $S(t) v=w$ for some $v$ in $V$;

particularly, if we suppose there exists a $J$-evolution process $T$ having the property

$$
S(t) T(t) v=T(t) S(t) v=v, \quad t \in J, v \in V,
$$

condition (viii) holds if $T$ is continuous at the left (right) with respect to its first argument and in the usual sense with respect to the second argument [that is, $\left(t_{n}\right.$; $n \in \mathbf{N}$ ) increasing (decreasing) in $J, t_{n} \rightarrow t \in J,\left(v_{n} ; n \in \mathbf{N}\right)$ in $V$ and $v_{n} \rightarrow v \in V$ imply $T\left(t_{n}\right) v_{n} \rightarrow T(t) v$ ]. Moreover, still assuming (vii) is accepted, it is also clear that a sufficient condition for (iii)' is

if the increasing (decreasing) sequence $\left(t_{n} ; n \in \mathbf{N}\right)$ in $J$ with (vii)' $\quad t_{n} \rightarrow t \in J$ and the sequence $\left(v_{n} ; n \in \mathrm{N}\right)$ in $V$ with $v_{n} \rightarrow v \in V$ are such that $S\left(t_{n}\right) v_{n} \rightarrow w \in V$ then $S(t) v=w$ 
a condition that may be interpreted as an order-closedness property of the $J$-evolution process $S$; note that, in such a situation, the corresponding variant of the second main result is comparable with Theorem 2 of Turinici [21] proved by a similar method. Finally, supposing in what follows $J=\mathbf{R}_{+}$, let $\varphi, \psi$ be a couple of functions from $\mathbf{R}_{+}$into itself. We shall say the $J$-evolution process $S$ is a $(\varphi, \psi)$-quasi-semigroup in case it satisfies the "functional" translation property

$$
d(S(t) u, S(s) v) \geqslant \varphi(s) d(S(\psi(t-s)) u, v), \quad t \geqslant s \geqslant 0, u, v \in V
$$

also, let us term the function $h$ from $\mathbf{R}_{+}$to itself, $\varphi$-admissible provided that

$$
\varphi(s) h(t-s) \leqslant h(t)-h(s), \quad t \geqslant s \geqslant 0 .
$$

Now, it is a simple exercise to verify that, in case the "decreasing" part of (iii) (respectively, (iii)') would be accepted, the couple of conditions

(ix) for every $s>0$ not belonging to $A$, we have

$$
\liminf _{t \rightarrow 0} \frac{d\left(S(\psi(t)) v, F_{s-t}\right)}{h(t)} \leqslant 1, \quad v \in F_{s},
$$

(x) for every $s>0$ belonging to A, we have

$$
\liminf _{t \rightarrow 0} \varphi(s-t) d\left(S(\psi(t)) v, F_{s-t}\right) \leqslant h(s)-h(s-0), \quad v \in F_{s},
$$

[respectively

(ix)' for every $s>0$ not belonging to $A$, we have

$$
\liminf _{t \rightarrow 0} \frac{d\left(S(\psi(t)) v, F_{s-t}(v, k(s)-k(s-t))\right)}{h(t)} \leqslant 1, \quad v \in F_{s},
$$

$(\mathrm{x})^{\prime}$ for every $s>0$ belonging to $A$, we have

$$
\begin{array}{r}
\liminf _{t \rightarrow 0} \varphi(s-t) d\left(S(\psi(t)) v, F_{s-t}(v, k(s)-k(s-t))\right) \leqslant h(s)-h(s-0), \\
\left.v \in F_{s}\right]
\end{array}
$$

appears as a sufficient one for the validity of the "decreasing" part of the couple (iv) + (v) (respectively, (iv)' $+(\mathrm{v})^{\prime}$ ); of course, when the $\mathbf{R}_{+}-$family $F$ is a constant one, the corresponding statement of these assumptions may be viewed as a quasi-semigroup counterpart of the "normality" condition used in Theorem 2 of Brézis and Browder [5] (see also Turinici [22]). Concerning this last aspect, it is not without importance to point out that, in case $\psi(t)=t, t \in \mathbf{R}_{+}$, an effective "time-dependent" extension of the above quoted results may be obtained along 
the following lines. Suppose the decreasing part of (iii) is substituted by

if the decreasing sequence $\left(t_{n} ; n \in \mathbf{N}\right)$ in $\mathbf{R}_{+}$with $t_{n} \rightarrow t \in \mathbf{R}_{+}$ and the sequence $\left(v_{n} ; n \in \mathbf{N}\right)$ in $V$ with $v_{n} \in F\left(t_{n}\right), n \in \mathbf{N}$, are such that $d\left(S\left(t_{n}-t_{m}\right) v_{n}, v_{m}\right) \leqslant \gamma h\left(t_{n}-t_{m}\right), n \leqslant m$, for some $\gamma>1$, then a $v \in F_{t}$ exists with $d\left(S\left(t_{n}-t\right) v_{n}, v\right) \leqslant \gamma h\left(t_{n}-t\right)$, $n \in \mathbf{N}$

then, under a normality condition similar in essence to (ix) we necessarily have the "translated" attraction property

$$
D_{2}\left(F_{a}, S(b-a) F_{b}\right) \leqslant h(b-a), \quad a, b \in \mathbf{R}_{+}, a<b .
$$

The essential idea of the proof is that of introducing a pseudo-metric and quasi-ordering structure on $X$ by the conventions

$$
\begin{aligned}
& e((t, u),(s, v))=|t-s|, \quad(t, u),(s, v) \in X, \\
& (t, u) \leqslant(s, v) \quad \text { if and only if } t \geqslant s \text { and } d(s(t-s) u, v) \leqslant \gamma h(t-s),
\end{aligned}
$$

and to apply Theorem 1 to its segment-subsets $Y$; a detailed exposition of the argument will be given in a forthcoming paper.

\section{Applications}

Let $(V, d)$ be a complete pseudo-metric space and $J$ a real interval. In what follows, by a multivalued mapping from $J$ into $V$ we shall mean any $J$-family in $V$ introduced as in Section 2. Now, given the function $h: J \rightarrow \mathbf{R}$, we shall say the multivalued mapping $T$ from $J$ into $V$ possesses a $D_{1}\left(D_{2}\right)$-mean value property with respect to $h$, provided that

$$
\begin{gathered}
D_{1}(T a, T b) \leqslant h(b)-h(a), \quad a, b \in J, a<b, \\
\left(D_{2}(T a, T b) \leqslant h(b)-h(a), \quad a, b \in J, a<b\right) .
\end{gathered}
$$

As already pointed out in the introductory part of the paper, the basic problem is that of determining sufficient local conditions in order that such a global property be obtained. In this direction, as a straightforward consequence of the first main result, we have

THEOREM 5. Suppose the order-usc multivalued mapping $T$ from $J$ into $V$ and the strictly increasing function $h: J \rightarrow \mathbf{R}$ are such that (iv) $+(\mathrm{v})$ are fulfilled with $S(t) v=v, t \in J, v \in V$. Then, $T$ possesses a $D_{1}\left(D_{2}\right)$-mean value property with respect to $h$, in the sense of (5). 
Let $(V, d)$ and $J$ be as before. Given the $i$-normal ( $d$-normal) multivalued mapping $T$ from $J$ into $V$ and the $i$-admissible ( $d$-admissible) function $k: J \rightarrow \mathbf{R}$ we shall say $T$ has a $k$-sectional $D_{1}\left(D_{2}\right)$-mean value property with respect to $h$ : $J \rightarrow \mathbf{R}$, provided that

$$
\begin{gathered}
D_{1}(T a, T b(T a, k(b)-k(a))) \leqslant h(b)-h(a), \quad a, b \in J, a<b, \\
\left(D_{2}(T a(T b, k(b)-k(a)), T b) \leqslant h(b)-h(a), \quad a, b \in J, a<b\right) .
\end{gathered}
$$

Now, as an immediate consequence of the second main result, we get

THEOREM 6. Let the order-usc i-normal (d-normal) multivalued mapping $T$ from $J$ into $V$, the i-admissible (d-admissible) function $k: J \rightarrow \mathbf{R}$ and the strictly increasing function $h: J \rightarrow \mathbf{R}$ be such that (iv)' $+(\mathrm{v})^{\prime}$ hold with $S(t) v=v, t \in J, v \in V$. Then, $T$ has a $k$-sectional $D_{1}\left(D_{2}\right)$-mean value property with respect to $h$, in the sense of $(5)^{\prime}$.

At this point, let us remember that-as already underlined in the preceding section-the basic hypothesis (iv) ((iv)') of the first (second) main result may be expressed under the symmetric form (vi) ((vi)') with respect to its component elements; this fact, combined with a univalence assumption about our mapping leads us-via Theorem 4-to the following extension of the well-known Dieudonné's mean value theorem [11, Ch. VIII, §5], [3, Ch. I, §3] (see also Turinici [26])

THEOREM 7. Suppose the mapping $T: J \rightarrow V$ satisfies an order-closedness property of the form

(vii)' for any increasing (decreasing) sequence $\left(t_{n} ; n \in \mathbf{N}\right)$ in $J$ with
$t_{n} \rightarrow t \in J$ and $T t_{n} \rightarrow v \in V$ we have $T t=v$

and let the increasing function $h: J \rightarrow \mathbf{R}$ be such that, a denumerable subset $A$ of $\mathbf{R}$ may be found with

(xii) for any s in $J \backslash A$ not identical with the eventual right (left) end-point of $J$ we have

$$
\begin{aligned}
& +\infty \neq \lim \sup _{r \rightarrow s+} \text { inf } \frac{d(T s, T r)}{(r-s)} \leqslant D_{(+)}^{+} h(s), \\
& \left(+\infty \neq \limsup / \inf \frac{d(T s, T r)}{(s-r)} \leqslant D_{(-)}^{-} h(s)\right),
\end{aligned}
$$

(xiii) for any $s$ in $J \cap A$ not identical with the eventual right (left) end-point of $J$ 
we have

$$
\begin{aligned}
& \liminf _{r \rightarrow s+} d(T s, T r) \leqslant h(s+0)-h(s), \\
& \left(\liminf _{r \rightarrow s^{-}} d(T s, T r) \leqslant h(s)-h(s-0)\right) .
\end{aligned}
$$

Then, necessarily, $T$ possesses a mean value property with respect to $h$ in the sense of (5).

Now, returning to the multivalued framework, suppose the pseudo-metric structure on $V$ is generated by a norm $\|\cdot\|$ with respect to which $V$ appears as a Banach space. Let $[a, b]$ denote (for any pair $a, b \in V, a \neq b$ ) the linear segment between $a$ and $b$ (that is, the image of the segment $I=[0,1]$ under the mapping $t \mapsto x_{t}=a+t(b-a)$ from $I$ to $\left.V\right)$ metrized and ordered by the standard procedure and put also $(a, b)=[a, b] \backslash\{a, b\}$. Given a multivalued mapping $T$ from $V$ into itself (equivalently, a mapping $T$ from $V$ into $2^{V}$ ) and a function $h$ : $\mathbf{R}_{+} \rightarrow \mathbf{R}_{+}$with $h(0)=0$, we shall say $T$ is $D$-lipschitzian with respect to $h$ provided that

$$
D(T x, T y) \leqslant h(\|x-y\|), \quad x, y \in V .
$$

The problem of determining a number of sufficient conditions (of a "segmentlocal" type) in order that such a global property be obtained will be referred to-after a terminology introduced by Kirk and Ray [16]-as a lipschitzianness test concerning the couple $(T, h)$. In this direction, as an immediate application of the preceding developments, the following lipschitzianness test may be formulated.

THEOREM 8. Let the multivalued mapping $T$ from $V$ into itself and the increasing function $h: \mathbf{R}_{+} \rightarrow \mathbf{R}_{+}$with $h(0)=0$ be such that, for any pair $a, b \in V, a \neq b$, the "combined" order-closedness property is fulfilled.

for any increasing or decreasing sequence $\left(x_{n} ; n \in \mathbf{N}\right)$ in $[a, b]$

(xiv) with $x_{n} \rightarrow x \in[a, b]$ and any sequence $\left(v_{n} ; n \in \mathrm{N}\right)$ in $V$ with

$v_{n} \rightarrow v \in V$, relations $v_{n} \in T x_{n}, n \in \mathbf{N}$, imply $v \in T x$

and also, that a couple of denumerable subsets $A_{a, b}$ and $B_{a, b}$ in $V$ may be found with

(xv) for any $x$ in $[a, b] \backslash A_{a, b}$ distinct from $b$,

$$
+\infty \neq \limsup _{(x, b) \ni y \rightarrow x} \frac{d(v, T y)}{\|x-y\|} \leqslant D_{(+)}^{+} h(\|x-a\|), \quad v \in T x,
$$

and for any $x$ in $[a, b] \backslash B_{a, b}$ distinct from $a$,

$$
+\infty \neq \limsup _{(a, x) \ni y \rightarrow x} \frac{d(v, T y)}{\|x-y\|} \leqslant D_{(-)}^{-} h(\|x-a\|), \quad v \in T x,
$$


(xvi) for any $x$ in $[a, b] \cap A_{a, b}$ distinct from $b$,

$$
\liminf _{(x, b) \ni y \rightarrow x} d(v, T y) \leqslant h(\|x-a\|+0)-h(\|x-a\|), \quad v \in T x,
$$

and for any $x$ in $[a, b] \cap B_{a, b}$ distinct from $a$,

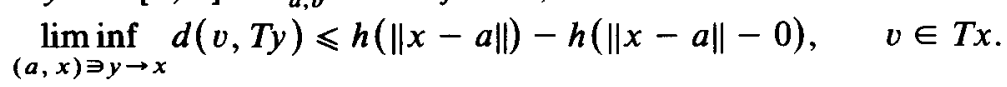

Then, $T$ is D-Lipschitzian with respect to $h$, in the above sense.

Proof. Let the multivalued mapping $T_{a, b}$ from $I$ into $V$ and the increasing function $h_{a, b}: I \rightarrow \mathbf{R}$ be defined as

$$
T_{a, b}(t)=T\left(x_{t}\right), \quad h_{a, b}(t)=h\left(\left\|x_{t}-a\right\|\right), \quad t \in I .
$$

It is a simple exercise to verify that, in view of (xiv)-(xvi), all conditions appearing in Theorem 4 are satisfied and the proof is complete.

As a first remark about this result, it must be noted that, under some smoothness assumptions about the couple $(T, h)$, the above statement provides us with the local variant of the multivalued fixed point theorem established by the author [23] (see also Nadler [18]). Secondly, under the assumptions $T$ is univalent and $h$ is linear, Theorem 8 clearly reduces to the Kirk-Ray lipschitzianness test we already quoted (see also Altman [1] or Turinici [24]). Finally, it is not without interest to emphasize that, by the use of an argument similar to that exposed here one may put this result into a metrically convex or, even an "abstract" metric framework, extending in this way some related contributions due to Husain and Sehgal [13] in the multivalued case, and respectively, to Clarke [10] (see also Kirk and Ray [17]) in the univalued case; some aspects of this problem will be discussed elsewhere.

\section{Acknowledgement}

The author is indebted to the referee for a number of valuable suggestions.

\section{References}

[1] M. Altman, 'Contractor directions, directional contractors and directional contractions for solving equations', Pacific J. Math. 62, 1-18, (1976).

[2] N. P. Bhatia and G. P. Szegö, Stability theory of dynamical systems, (Springer-Verlag, Berlin, 1970).

[3] N. Bourbaki, Eléments de mathématiques. Livre IV (Fonctions d'une variable réele), Chs. I-III, 2ième ed. (Hermann, Paris, 1958). 
[4] H. Brézis, 'On a characterization of flow-invariant sets', Comm. Pure Appl. Math. 23, 261-263, (1970).

[5] H. Brézis and F. E. Browder, 'A general principle on ordered sets in nonlinear functional analysis', Advances in Math, 21, 355-365, (1976).

[6] A. Brondsted, 'On a lemma of Bishop and Phelps', Pacific J. Math. 55, 335-341, (1974).

[7] A. Brondsted, 'Fixed points and partial orders', Proc. Amer. Math. Soc. 60, 365-366, (1976).

[8] F. E. Browder, 'On a theorem of Caristi and Kirk', Fixed point theory and its applications, pp. 23-27 (Academic Press, New York, 1976).

[9] J. Caristi, 'Fixed point theorems for mapping satisfying inwardness conditions', Trans. Amer. Math. Soc. 215, 241-251, (1976).

[10] F. H. Clarke, 'Pointwise contraction criteria' for the existence of fixed points', Canad. Math. Bull. 21, 7-11, (1978).

[11] J. Dieudonne, Foundations of modern analysis, (Academic Press, New York, 1960).

[12] I. Ekeland, 'Nonconvex minimization problems', Bull. Amer. Math. Soc. (N.S.) 1, 443-474, (1979).

[13] S. A. Husain and V. M. Sehgal, 'A remark on a fixed point theorem of Caristi', Math. Japonica 25, 27-30, (1980).

[14] S. Kasahara, 'On fixed points in partially ordered sets and Kirk-Caristi theorem', Math. Sem. Notes Kobe Univ. 3, 229-232, (1975).

[15] W. A. Kirk, 'Caristi's fixed point theorem and metric convexity', Colloq. Math. 36, 81-86, (1976).

[16] W. A. Kirk and W. O. Ray, 'A remark on directional contractions', Proc. Amer. Math. Soc. 66, 279-283, (1977).

[17] W. A. Kirk and W. O. Ray, 'A note on Lipschitzian mappings on convex metric spaces', Canad. Math. Bull. 20, 463-466, (1977).

[18] S. B. Nadler, Jr., 'Multivalued contraction mappings', Pacific J. Math. 30, 475-488, (1969).

[19] L. Pasicki, 'A short proof of the Caristi theorem', Comment. Math. Univ. Carolin. 20, 427-428, (1978).

[20] J. Siegel, 'A new proof of Caristi's fixed point theorem', Proc. Amer. Math. Soc. 66, 54-56, (1977).

[21] M. Turinici, 'Flow-invariance theorems via maximal element techniques', Nederl. Akad. Wetensch. Proc. Ser. A 84, 445-457, (1981).

[22] M. Turinici, 'Stability criteria for contractive semigroups via maximality procedures', Bull. A ustral. Math. Soc. 24, 453-469, (1981).

[23] M. Turinici, 'Multivalued contractions and applications to functional differential equations', Acta Math. Acad. Sci. Hungar. 37, 147-151, (1981).

[24] M. Turinici, 'Function Lipschitzian mappings on Banach spaces and applications', Rev. Roum. Math. Pures Appl. 27, 211-217, (1982).

[25] M. Turinici, 'Constant and variable drop theorems on metrizable locally convex spaces', Comment. Math. Univ. Carolin. 23, 383-398, (1982).

[26] M. Turinici, 'Mean value theorems via maximal element techniques', J. Math. Anal. Appl. 88, 48-60, (1982).

[27] M. Turinici, 'Differential inequalities on abstract metric spaces', Funkcial.Ekvac. 25, 227-242, (1982).

[28] C. S. Wong, 'On a fixed point theorem of contractive type', Proc. Amer. Math. Soc. 57, 283-284, (1976).

Seminarul Matematic "Al.Myller"

University of Iași

6600 Iaşi

Romania 